\title{
Time-Reversal Invariance and Thermodynamics of Electromagnetic Fields in Materials with Memory.
}

\author{
Rinaldo Borghesani - Afgelo Morro (Genova) (*)
}

When our paper [1] was in press, we became aware of two very recent contributions by Rivuin [2] and by Meixner [3], where two definitions of time-reversed functions are assumed. In order to avoid possible misinterpretations of some of our results, we show that our main theorems maintain essentially their validity even if the definitions of [2], [3] are adopted.

Following closely the notation given in [1], we recall our unique definition of the time-reversal of a closed path. If $f(\cdot):(-, \infty, \infty) \rightarrow \mathcal{V}$, the time-reversed path $\tilde{f}(\cdot):(-\infty, \infty) \rightarrow \vartheta$ is defined by

$$
\tilde{f}(t)=f(-t) .
$$

According to $[2],[3]$ we are led to lay down two distinct definitions which have only a microscopical justification-see the references quoted in [1]- More precisely, if $f_{1}$ is a polar vector, the closed path $f_{1}(\cdot):(-\infty, \infty) \rightarrow \mho$ has the following time-reversed path $\tilde{f}_{1}(\cdot):(-\infty, \infty) \rightarrow \mathcal{Y}$ such that

$$
\tilde{f}_{1}(t)=f_{1}(-t)
$$

On the contrary, when $f_{2}$ is an axial vector, the time-reversed $\tilde{f}_{2}(\cdot):(-\infty, \infty) \rightarrow$ U is given by

$$
\tilde{f}_{2}(t)=-f_{2}(-t)
$$

If the above definitions are taken into account, the 2nd theorem of [1] suffers very slight modifications. In fact, when $\boldsymbol{u}(\cdot)$ and $\boldsymbol{v}(\cdot)$ are derived from a polar and from an axial vector respectively, then our (2.10) reads

$$
\mathcal{G}(\boldsymbol{u}, \boldsymbol{v})-\boldsymbol{S}(\tilde{\boldsymbol{u}}, \tilde{\boldsymbol{v}})=\int_{-\infty}^{\infty} \int_{-\infty}^{t}\left[\boldsymbol{u}(t) \cdot \boldsymbol{M}(t-s) \boldsymbol{v}(s)+\boldsymbol{v}(t) \cdot \boldsymbol{M}^{\boldsymbol{T}}(t-s) \boldsymbol{u}(s)\right] d s d t
$$

where $\boldsymbol{M}=\boldsymbol{A}_{1}+\boldsymbol{A}_{\mathbf{2}}^{\boldsymbol{r}}$. Obviously the conclusion $\boldsymbol{M}(s)=\mathbf{0}, \forall s \geqslant 0$ holds again. Also

(*) Entrata in Redazione il 12 luglio 1974. 
for the 3 th theorem of [1] irrelevant changes are necessary. If $\boldsymbol{u}$ is an axial vector, a direct inspection provides

$$
\boldsymbol{B}(s)=\mathbf{0} \quad \text { and } \quad \boldsymbol{D}(s)=\mathbf{0} \quad \forall s \geqslant 0
$$

In conclusion, the only modification in the statement of our main 6th theorem is: $C_{\mathrm{IV}}=\mathbf{0}$; thus we have

$$
\begin{aligned}
& e_{1}=C_{\mathrm{I}} * \theta+\boldsymbol{C}_{\mathrm{II}}^{0} * \mathbf{g}+\boldsymbol{C}_{\mathrm{III}}^{0} * \boldsymbol{E}, \\
& \boldsymbol{q}_{1}=\boldsymbol{K}_{\mathrm{II}} * \boldsymbol{g}+\boldsymbol{K}_{\mathrm{III}} * \boldsymbol{E}, \\
& \boldsymbol{j}_{1}=\boldsymbol{\Sigma}_{\mathrm{II}} * \mathbf{g}+\boldsymbol{\Sigma}_{\mathrm{III}} * \boldsymbol{E},
\end{aligned}
$$

with $\boldsymbol{K}_{\mathrm{II}}=\boldsymbol{K}_{\mathrm{II}}^{T}, \boldsymbol{\Sigma}_{\mathrm{III}}=\boldsymbol{\Sigma}_{\mathrm{III}}^{T}$ and $\boldsymbol{K}_{\mathrm{III}}+\theta_{0} \boldsymbol{\Sigma}_{\mathrm{II}}^{T}=\mathbf{0}$. Of course the relaxation property for $e$ ( 7 th theorem) yields $C_{\mathrm{xI}}^{0}=\mathbf{0}$ and $C_{\mathrm{III}}^{0}=\mathbf{0}$. It is worthwhile to remark that in our analysis, both with a unique and with two distinct definitions of time-reversal, we derive only Onsager-type symmetry relations. The absence of Casimir-type antisymmetry properties is a consequence of the form of our entropy production which cannot be rearranged in the classical form examined by RIVLIN [2].

\section{REFERENCES}

[1] R. BoRghesani - A. MoRRo, Time-reversal invariance and thermodynamics of electromagnelie fields in materials with memory, Ann. Mat. Pura Appl., IC (1974), pp. 65-80.

[2] R. S. Rrvun, A note on the Onsager-Casimir relations, Zeit. angew. Math. Phys., 24 (1973), pp. 897.899 .

[3] J. MeIXner, Macroscopic and microscopic reversibility, private communication. 